\title{
Perceptions of physiotherapists of their role in reducing pain and increasing, function, strength and flexibility in patients with Patellofemoral Pain Syndrome
}

\author{
Konstantinos Papadopoulos ${ }^{1 *}$, Russell Kabir ${ }^{2}$ and Dimitris Stasinopoulos ${ }^{3}$
}

*Correspondence: kostpap@yahoo.com

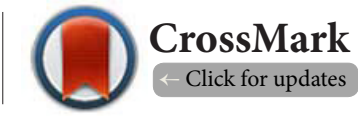

${ }^{1}$ Middlesex University London, Faculty of Science and Technology, The Burroughs, Hendon, London NW4 4BT, UK.

${ }^{2}$ Anglia Ruskin University, Public Health Department, Faculty of Medical Sciences, UK.

${ }^{3}$ Physiotherapy programme, European University Cyprus, Nicosia, Cyprus, 1516, UK.

\begin{abstract}
Study objectives: The purpose of this focus group study was to establish the physiotherapy treatment of Patellofemoral Pain Syndrome in North-West Wales. In addition the study aimed to report the barriers that stopped physiotherapists from increasing strength and flexibility and the contradictions of physiotherapists' beliefs regarding their practice.

Methods: The investigation was based on specific and priori designed questions. Two focus groups were conducted, where physiotherapists discussed the results of a feasibility study conducted in their department. 11 hypotheses discussed whilst 13 evidence statements reported by the merger of the answers to the hypotheses. A level of consensus was described using the moderator's notes.

Results: Patellofemoral Pain Syndrome physiotherapy works; not through strength and flexibility but through pain and function improvement. However, this practice often only has a short-term effect.

Conclusions: Group classes and better education on the importance of specific exercises and self-managing should be researched whilst the long-term effect of these treatment components should also be assessed.

Keywords: Focus group, physiotherapy practice, patellofemoral pain syndrome, strength, flexibility, pain, function
\end{abstract}

\section{Introduction}

A previous study contacted at North-West Wales (NWW) National Health Service (NHS) physiotherapy departments reported that physiotherapists used a series of strength and flexibility tests to assess and treat PFPS [1]. This situation is in line with the regulations of the Health and Care Professions Council (HCPC) standards of physiotherapy proficiency which suggest the use of safe and effective physiotherapy-skills including exercise and movement [2]. However, a feasibility study conducted at the same physiotherapy departments of NWW which aimed to monitor the effectiveness of six-week period of physiotherapy performed on 26 patients with Patellofemoral Pain Syndrome (PFPS) showed that isometric strength and flexibility of several low limb muscles of patients with PFPS were not increased significantly [3] (appendix 1). On the other hand, their pain levels and function measured by two pain Visual Analogue Scales (VAS), and one function scale named Anterior Knee Pain Scale (AKPS), were improved significantly [3] (appendix 1). This was an unexpected finding and to investigate it further a focus group study was set up. The objective of this was to investigate what NWW physiotherapists, who treat NHS patients with PFPS, think about the findings of this study and how they might explain it. This study also aimed to identify whether there are any barriers which restrained physiotherapists from adopting the research evidence and what the implications of any modified practice were. If a modified intervention took place, the maintenance of intervention effects should also be considered. It was decided that a focus group study would be the most appropriate to investigate NWW physiotherapy practice relating to PFPS. 
Papadopoulos et al., Physical Therapy and Rehabilitation 2017,

http://www.hoajonline.com/journals/pdf/2055-2386-4-9.pdf

doi: 10.7243/2055-2386-4-9

\section{Methods}

Kitzinger's methodology was used for this focus group study [4]. This methodology is particularly useful for exploring people's knowledge and experiences and can be used to examine not only what people think but how they think and why they think that way [4]. The advantage of focus groups lies in the fact that they can encourage participation from those who are reluctant to be interviewed on their own and that they allow comparison of individual opinions after interactive discussion [4]. In addition, it was hoped that it would be possible to come to a consensus regarding PFPS physiotherapy practice and this could only come about if those who deliver physiotherapy practice participated in the study. Consensus was achieved by using Onwuegbuzie's principles [5].

The Ritchie and Spencer (1994) method of qualitative data analysis was selected because this framework was developed specifically for more applied qualitative research and it has an appeal to all those working in public health and related fields. This framework analysis is particularly appropriate when a study has clear aims at the outset.

In this focus group study, the findings of previous studies contacted in the same clinical departments were used and their application and 'truth value' were explored with NHS physiotherapists who treat PFPS.

\section{Aims and research questions}

The aim of this study was to establish NWW physiotherapy treatment of PFPS and report the barriers that stopped physiotherapists from increasing strength and flexibility and the contradictions of physiotherapists' beliefs regarding their PFPS practice. The investigation was based on specific and priori designed questions (Figure 1).

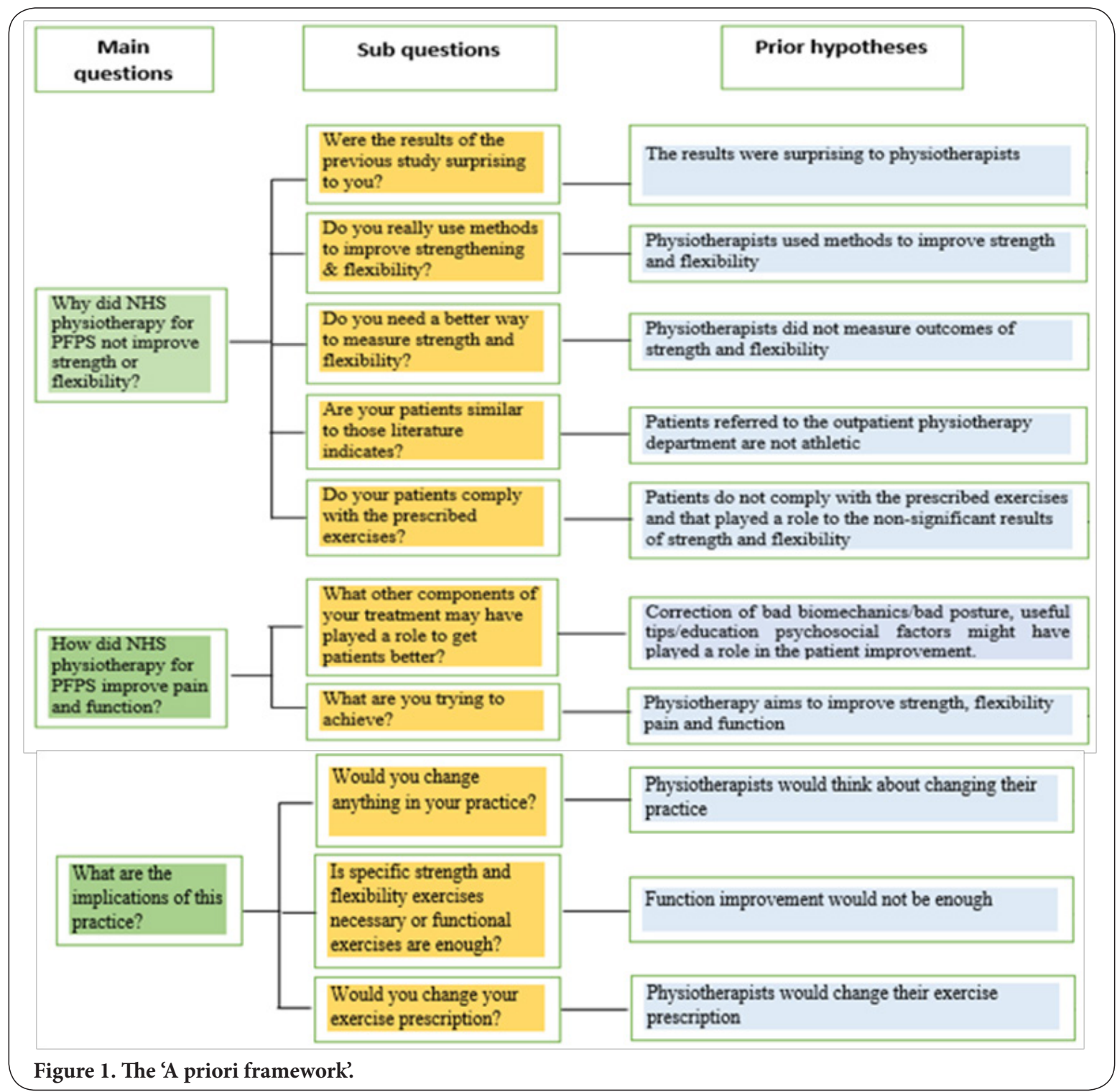


Papadopoulos et al., Physical Therapy and Rehabilitation 2017,

http://www.hoajonline.com/journals/pdf/2055-2386-4-9.pdf

\section{Ethical approval}

Ethical approval was sought from the Ethics Committee of the School of Sports Health and Exercise Sciences, Bangor University, whilst, research and development (R\&D) approval was obtained by the Betsi Cadwaladr University Health Board. Written participant information sheets were given to all physiotherapists at least 24 hours before they decided whether to take part in this study or not. Consent forms were signed by physiotherapists on the day of their participation. All information collected about participants was kept strictly confidential. Any information about the participants had their name removed and were identified by a number so they could not be recognised from it. Participants' personal details were available only to the researcher. The participants received a five pound High Street voucher after the focus group study was completed.

\section{Recruitment/participants}

Physiotherapists at the local hospital who had treated patients with PFPS were asked to participate in this study. An Extended Scope (ES) physiotherapist (MB) did the recruitment by informing her colleagues about the study and by giving them the participant information sheet. A few dates were proposed as the most feasible for the focus groups. The physiotherapists informed the ES physiotherapist about which of the proposed dates suited them better. The dates with the most available physiotherapists were set as focus group days.

\section{Data collection}

Ideally, focus groups should have between 4 and 8 participants in order to facilitate interaction and discussion between participants to explore specific issues or topic of interest [6]. Two separate focus groups were planned in the physiotherapy department. The first focus group was performed at 9am UK time and included five physiotherapists. The second was performed at $1 \mathrm{pm}$ (same day with the first focus group and same time zone) and included seven physiotherapists. The first group had more years of physiotherapy practice (13.8 \pm 8.8 years) than the second group ( $8.2 \pm 4.3$ years). Table 1 shows the characteristics of the physiotherapists who took part in this study.

The duration of the focus groups was between 45 and 70 minutes long. In order to get the most of the interviews and to achieve a good quality of sound a quiet room was chosen. Participants were asked to sit in a circle. Both focus groups had a moderator and an assistant moderator with previous experience in qualitative interviews. According to Krueger [7] it is ideal for the focus group to have a'moderator'. The 'moderator' was responsible for facilitating the discussion, prompting members to speak, requesting overly talkative members to let others talk and encouraging all members to participate. This happened in a non-directive and unbiased way. The 'assistant moderator' was used as a scribe who wrote down the participants' reaction to their colleagues' responses
Table 1. Physiotherapists' characteristics of the two focus groups.

\begin{tabular}{lll}
\hline Focus group 1 & Sex & Years of practice \\
\hline P1 & Female & 20 years \\
P2 & Female & 20 years \\
P3 & Male & 8 years \\
P4 & Female & 1 years \\
P5 & Female & 20 years \\
\hline Focus group 2 & Sex & Years of practice \\
\hline P1 & Female & 10 years \\
P2 & Female & 5 years \\
P3 & Female & 6 years \\
P4 & Male & 10 years \\
P5 & Male & 10 years \\
P6 & Female & 2 years \\
P7 & Female & 15 years \\
\hline
\end{tabular}

on a large sheet of paper. The 'moderator'was given prompt questions (questions and sub questions) that the researcher was planning to use beforehand. For each question, the moderator reported a positive or negative expression for all participants according to what they said or their body language (nods or shakes of the head for 'no' or other small verbal or facial expressions) [8]. The reason for having an'assistant moderator' to facilitate this study was to measure the level of consensus in the answers physiotherapists gave.

Before discussions started participants were provided with two supplemental documents which included one figure and one table (appendix 1 and 2). These documents reported the major results of the effect of their treatment regarding strength, flexibility, pain and function in patellofemoral pain syndrome (PFPS) patients as found in a feasibility study conducted in their NHS physiotherapy department (appendix 1). The figure was used to remind the physiotherapists the methods they said they use when treating patients with PFPS (appendix 2). After this, the participants were asked to discuss the major questions and sub questions of the a priori framework.

\section{Data analysis}

Audio-taped interviews were anonymised, transcribed verbatim and uploaded into the computer software package Atlas. ti, version 6.1.1 (GmbH, Berlin) to organise, analyse and sort data. Data were analysed independently by two researchers. The analysis was subjected to the framework analysis of Ritchie and Spencer [9].

Ritchie and Spencer [9] described five stages in framework analysis. The first step was familiarisation. As the focus group study was designed to bring together findings from previous studies in the thesis, the process of familiarisation involved making explicit previous study and the review of publications findings that warranted further explanation and drawing out a set of questions and hypotheses to be explored and tested in focus groups with physiotherapists. The second step was 
identifying a priori framework of questions, sub questions and hypotheses to guide interpretation of focus group evidence (see Figure 1). The third step was indexing. In this stage the a priori framework was applied to the whole focus group data set. To enhance scientific rigour, two review authors (KP, RK) read and re-read the transcripts and applied the priori framework moving back and forth between the data and the framework and searching for evidence linking with each hypothesis [10], which was then discussed with both researchers. The boundaries of the emerging explanations and complexity between the explanations were discussed among the authors. The fourth stage was charting. In this stage, data were rearranged in charts to align related evidence with specific hypotheses. Finally, the fifth stage was Mapping and interpretation. Evidence was mapped against specific hypotheses and used to support or refute and where appropriate seek out varying explanations.

The moderator was asked to keep notes according to the responses to the prompt questions. Consensus was considered as high when 11 or 12 (all), moderate when $8-10$, and low when 1-8 physiotherapists expressed the same opinion. Additionally, factors including frequency, emotional expression and extensiveness of the comments were also considered during the process $[11,12]$. The moderator was also asked to add any other issues discussed during the interviews and the way physiotherapists responded to them [13]. This helped in identifying explanations and ideas discussed during the interviews relevant to the issue of why physiotherapists did not increase strength and flexibility while they improved function and pain.

\section{Results}

Hypothesis that the results were surprising to physiotherapists

Physiotherapists were asked to discuss the finding that a sixweek course of physiotherapy did not increase strength and flexibility in PFPS patients. This finding was not in line with the reported physiotherapy treatment objectives, according to which, they aim to get patients stronger and more flexible (appendix 2). However, the hypothesis was rejected because the physiotherapists did not appear to be surprised by the results. The main reason given was there was not enough time to physically increase strength and flexibility. Participants believed that longer than six weeks was required to increase muscle strength while increased flexibility would not occur before the muscles gained other characteristics such as control. These are illustrated by the following quotes:

\section{Strength}

FG2P3:...I was not surprised that there was no change in strength. As much as I am concerned it takes more than 6 weeks to become stronger anyway.

FG1P5:.... and I think it takes more time to strengthen than 6 weeks. I know studies that have been done before, they have shown it could take more than a year to build up good strength back, in conditions like ACLs or something; it takes more time to build up muscle strength; at least to get an obvious difference that you could pick up statistically, so I think it might takes longer than just 6 weeks.

\section{Flexibility}

FG2P3:...maybe not enough time.

Researcher: not enough time from you or from the patients? FG2P3: For the muscles, to get some length...

FG2P6:...I think there is some evidence that some of the muscle are reducing strength and becoming tight because they are trying to stabilize joints because the muscles around them aren't controlling them. So, in six weeks if you give them flexibility exercises, and you have not increased the control of the muscles then you won't get any muscle length.

\section{Evidence statement 1}

Physiotherapists did not deny that their practice aimed to increase strength and flexibility. However, a six-week time period was not enough to demonstrate improvement in strength and flexibility. Additionally, they reported that if patients were measured later than those six weeks, those results would probably be significant. This shows that physiotherapists believed that their treatment has a long term effect which lasts even after patients are discharged from the physiotherapy department.

Hypothesis that physiotherapists used methods to improve strength and flexibility

Physiotherapists were asked whether they believed that patients with PFPS really became stronger and more flexible with treatment. Their answer was positive and the hypothesis seemed to be accepted (Figure 2). However, when discussion revealed how they increased strength and flexibility, it became apparent that what physiotherapist meant by increasing strength was not what physiologists/sport scientists mean. There was disagreement about how strengthening can be achieved, with physiotherapists believing strengthening is achieved by general functional exercises, whereas physiologist/sports scientists believe that an measured increase in strength can only be achieved specific intense exercises. The same issue appearedregarding flexibility. The physiotherapists appeared to be uncertain regarding the severity of exercise that is needed to be applied to gain strength or the stretching techniques required for a significant measurable muscle lengthening effect.

\section{Strength}

FG1P2: I think strengthening is what makes functional...I think to me strengthening is getting the muscle in a functional way.

FG1 P5:...I still think a lot of it, I mean there is an element to strength; a lot cannot just be that proprioceptive, I mean this is not necessarily condemned to strength... it is about the quality 
Papadopoulos et al., Physical Therapy and Rehabilitation 2017,

http://www.hoajonline.com/journals/pdf/2055-2386-4-9.pdf

doi: 10.7243/2055-2386-4-9

of movement and the finding that the firing of different muscle is there.

FG2P4: I give some people to do repetitions of sitting to stand in a proper alignment and that is functional but it strengthens as well.

\section{Flexibility}

FG1P5:...again, unless flexibility is actually changing the functional angle... it is probably not going to change that much...is it? FG1 P4: I might give them ITB stretch, like a small squat flattening back against the wall then try to hold it...like a squat... but again it is the quality of movement rather than...stretch... and core stability.

\section{Evidence statement 2}

NHS physiotherapists do not have the same perception as physiologists/sport scientists regarding how strength and flexibility can be achieved. Physiology evidence shows that an increase in muscle strength can be achieved in six weeks if intense and specific strength and flexibility programs are applied 3 times per week or in a day by day basis.

Hypothesis that physiotherapists did not measure outcomes of strength and flexibility

One of the key messages that physiotherapists reported in the past [1] was that North-West Wales (NWW) physiotherapists hardly use outcome measures in their practice. Therefore, the hypothesis was that physiotherapists did not increase strength and flexibility because they did not measure the outcomes of this practice. The hypothesis was appeared to be rejected in the beginning because physiotherapists agreed that it would be ideal to have an isokinetic dynamometer to measure strength or an electronic goniometer to measure flexibility. However, after some discussion the physiotherapists appeared to change their mind for two major reasons; the first was that such instruments would need a lot of time to be set up and this would waste what little time they have for each patient (20 minutes) and second, they realized that it is not a typical physiotherapy practice to measure these two muscle characteristics. Therefore, the hypothesis was accepted.

FG1 P3: ...If we had that (isokinetic dynamometer) with patients it would be great.

FG1P5: ....But it needs something to be simple. Like the grip strength with hands. So it has to be something very practical. To be realistic, the time we have with a patient is not enough for something complicating.

FG2P4:.... we do not measure strength, we do not have these results. That is not our normal practice. We do not aim to reach a specific amount of strength.

FG2P2: ...but we do measure muscle bulk don't we?

FG2P4: ...the thing is that when you measure muscle bulk your points might be different to mine... it is very subjective isn't it? We do not really measure it, do we? But if you had a measure to point 'this is where these patients are' this wouldn't depend on which physio does it because it would be in the same platform.

\section{Evidence statement 3}

It is not a typical NWW physiotherapy practice to measure strength and flexibility. This evidence shows that specific level of these two characteristics was not intended to be achieved.

\section{Hypothesis that patients referred to the outpatient} physiotherapy department are not athletic

A literature review [14] showed that most of the patients who take part in PFPS research studies are athletic. However, the hypothesis was that the patients physiotherapists see in the NHS clinic were not athletic since the mixed method study and the researcher's experience at a district hospital supported this opinion. When physiotherapists were asked whether their patients are similar to those the literature reports, they answered in the negative reporting that most of their patients are not athletic all. They reported that they see patients with different activity levels (from very active to no active at all). Their belief was that most of their patients have PFPS because they do not do any exercise rather than from overuse. In fact some of the physiotherapists reported that if their patients were active they would not need to consult to them. Therefore, the hypothesis was accepted.

FG1P2:... we think that $80 \%$ of them aren't and this is a problem we have to confront.

FG2P7:...they vary massively; you can get sport people with high level of motivation and people who do actually nothing.

FG1P3:...If they were active, would they be here? I do not know...

FG2P3:...the active ones might go private first because they would like to see a sport physio and they want to see someone quickly.

FG1P2:...In private practice you do not get to see these people (non-active patients) very often and they are keener to do what they are told...

\section{Evidence statement 4}

Physiotherapists have to deal with non-athletic patients. This kind of patients are not familiar with sport activities, thus physiotherapists prescribe simple and generic exercises (otherwise patients will not perform them) which may lead to a non-significant increase of strength and flexibility. This contradicts the first evidence statement according to which a longer time period would change patients' strength and flexibility.

Hypothesis that patients do not comply with the prescribed exercises and that played an important role to the non-significant results of strength and flexibility The hypothesis was that patients did not comply with the prescribed exercises and this was supported by a previous study [1] which found that physiotherapists believed that most of their patients would not comply with the prescribed exercises. The hypothesis was accepted as physiotherapists 


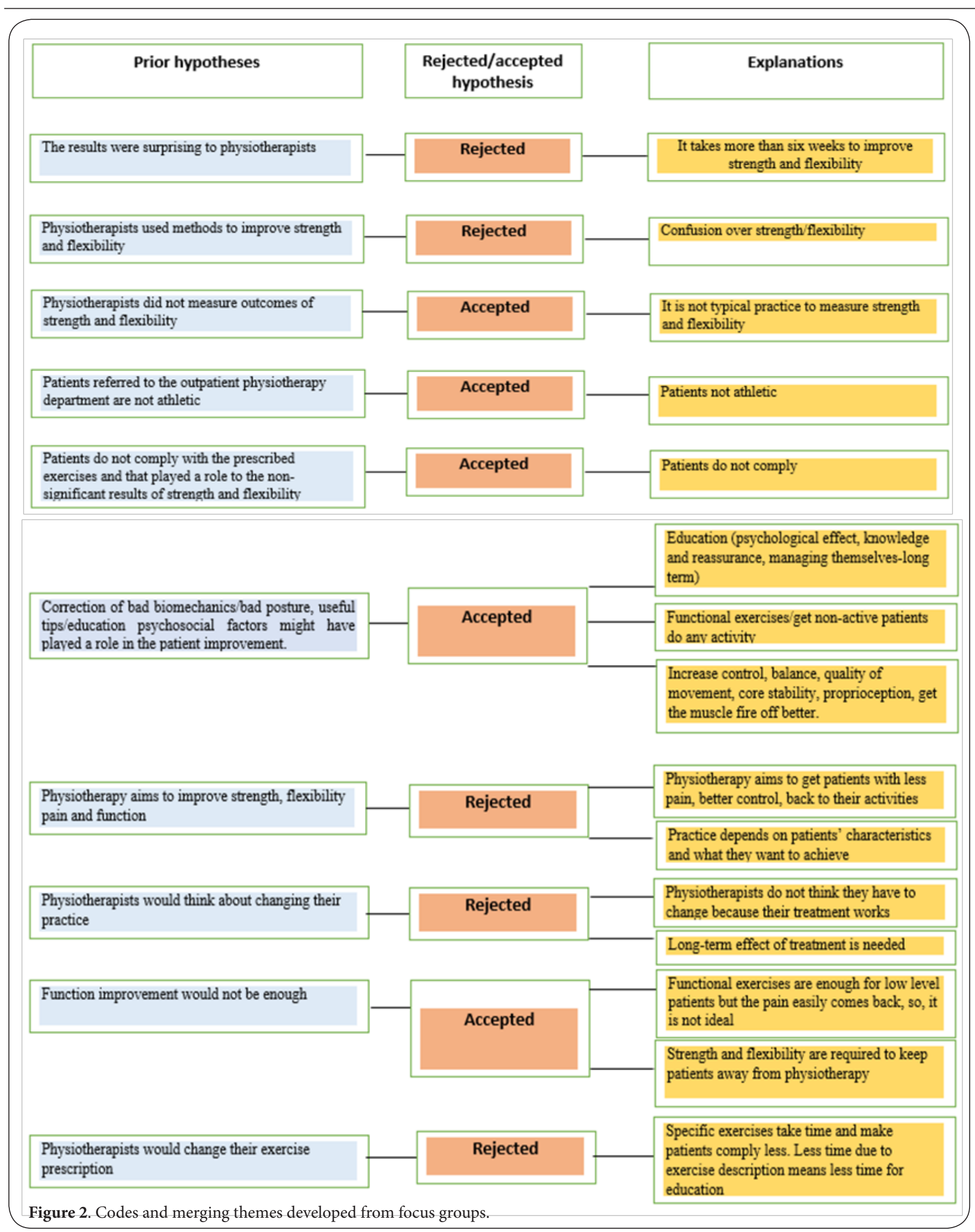


reported three major reasons; first, patients' lack of interest in doing exercises, second, their busy schedule and third, physiotherapy is at some cases an 'exit route', which means that physiotherapy can be a way to discharge patients from hospitals. Physiotherapists also reported that the fact that patients do not comply with their prescribed exercises has to do with patients' physical activity. Less active patients are unlikely to comply at all.

FG1P1:...It depends on whether they are used to exercising.

FG1P2:... well some of them may would, if they do not work

FG2P5:...people who want to get better and more active will tend to listen more, while those who are obese and not active are most likely to referred to the physiotherapy by a GP just because it is an exit route. These patients do not really want to be here...

FG2P7:...I think that even the athletic patients do not necessarily comply, some of them do some of them don't. I think the problem with these high activity level patients is that even if they do their exercises they do not stop their activity because they are determined to carry on.

\section{Evidence statement 5}

Patients' perceived lack of compliance with prescribed exercises affects physiotherapy practice by inducing physiotherapists to prescribe a few and simple exercises. This may lead to a non-significant increase of strength and flexibility at 6 weeks.

Hypothesis that correction of bad biomechanics/bad posture, useful tips/education psychosocial factors might have played a role in the patient improvement

The physiotherapistshave previously reported [1] that use a series of different treatment components when treating PFPS patients. The hypothesis was accepted. However, more treatment methods than expected were reported. The treatment methods could be separated in three major categories. The first two categories were the two major components of their treatment which included 1) education and 2) functional exercises/getting patients do any activity, while the third one included all the other biomechanical components entitled as: 3) Increase control, balance, quality of movement, core stability, proprioception, get the muscle fire off better. Evidence statements were created for each of the three components of their treatment separately.

\section{Education}

Education was reported as one of the most important treatment component achieving pain decrease. Education contained three different components (psychological effect, knowledge and reassurance and self-managing). Knowing why patients have to do the exercises can designate compliance.

\section{Psychological effect}

FG1P5:...yes it a sort of placebo effect as well.

FG1P3:...it is a psychological thing...you know, pain is felt in here (showing the head) rather than in here (showing the knee) so I think we cannot discount that coming to physio and being assured that everything is alright and that they just need to get moving, probably improves the function and pain.

\section{Knowledge and reassurance}

FG1P2:... We usually explain to patients why they have the pain... they understand why they are having the problem...because the muscles aren't firing in the right way, there is no balance... and we tell them 'let's get them work in the right way.'

FG1 P4:... some patients come and say 'oh I am glad I heard that, I was scared of doing exercises.... and then after a couple of weeks they come back and say: 'I do not have any knee pain anymore'.

\section{Self-managing}

FG1P1: ... also teach them to do their own patellofemoral moves so they can do their own stretches at home with that.

FG1 P5:... I think if you encourage them long term to do more activity that can keep it away for longer, otherwise they might come back very soon. You need to change their function long terms and encourage them to start going to the gym and build up strength and keep it for long period.

FG2P5:... if the patients know why they are doing it, it is more likely they comply. If they know that they are going to get some benefit from doing the exercises then they are going to do them if not then there is no chance.

\section{Evidence statement 6}

Education (psychological effect, knowledge and reassurance and self-managing) plays an important role in reducing patient's pain and improving function, enhancing patient compliance and keeping patients without pain long-term.

\section{Functional exercises/getting patient do any activity} Physiotherapists reported that they mainly prescribe functional exercises to patients (e.g. walking up and down the stairs correctly, controlled knee bends) and in some cases they only ask from their patients to get involved in any sport activity (gym, ball games) since their muscle tone is extremely low. This practice can also be linked with patients' reluctance to comply since they often are not athletic and not keen on doing specific exercises. However, this can also be another reason why strength and flexibility are not increased after a six-week treatment.

\section{Functional exercises for strength and flexibility}

FG1P2:...getting them to do normal everyday things, standing properly, and walking up and down the stairs, just normal functional things.

FG1P2:...for the calves we usually ask them to do some small knee bends, to get some small stretch when they are doing that... or the usual one of the edge of the step...for the hamstrings we usually ask them to do this..(flex their trunk over their legs) they also get to have the control of their trunk when we ask them to do that... 
Papadopoulos et al., Physical Therapy and Rehabilitation 2017,

http://www.hoajonline.com/journals/pdf/2055-2386-4-9.pdf

doi: 10.7243/2055-2386-4-9

FG1P3:...Similar... might give them hip flexors stretch when the gluts are weak...I give them hamstring too...strength wise I give quads closed chain exercises...

FG1P2: I think that they key is the function and this doesn't need any muscle length or muscle strengthening.

\section{Getting patients do any activity}

FG1P2:...yes sometimes you have to draw a line and think that... yes you are dealing with AKP but...the only thing you can do is to get them do ANY muscle working just to make any difference. FG1P5:...And I think with teenagers I go away from specific exercises; I just tell them to go to the gym to get some muscle tone. FG1P5:... and sometimes, I think with the older patients if you just encourage them to keep moving...because if you get them moving there is blood supply and this improves the pain anyway... so even if you get them do some sittings on the desk, this can make a difference, however you are not improving any of their muscle strength.

\section{Evidence statement 7}

Functional exercises/inducing patients to do any activity are suitable for the type of patients physiotherapists see in the clinic and play an important role in reducing patients' pain and improving function; however they do not increase strength and flexibility.

Increase control, balance, quality of movement, core stability, proprioception, get the muscle fire off better This hypothesis included all other components of physiotherapy treatment that decreased pain and increased function. Physiotherapists reported that they use them according to patients' needs. All patients do not necessarily need to get stronger and more flexible but they might need some other kind of improvement.

\section{Treatment components that increase function and de- crease pain}

FG1P5:...yeah the quality of the posture and movements and the quality of right muscle working at the right time.

FG1P2:...changing how they move and what muscles work.

FG2P6:...their muscle control as well...you do not need to have strength to control your muscles in a better way...control and maybe proprioception makes the difference as well.

FG1P4:...the balance as well.

FG2P6:....we also do proprioception, core stability, foot posture and other things as well, so, it is not that we are only looking at strength and flexibility.

FG2P7:...I think we assess any biomechanical issues anywhere around.

\section{Strength and flexibility is not always the case}

FG1P3:...you can have a young male/female with good strength and still have Anterior Knee Pain (AKP), so it might be a matter of just getting the quality better...

\section{Evidence statement 8}

A variety of different treatment modalities are used to increase function and decrease pain. They are all patient-dependent. Focusing on these components rather than active treatments may have played an important role in not increasing strength and flexibility however; all patients do not need better strength and flexibility.

Hypothesis that physiotherapy aims to improve strength, flexibility pain and function

A previous study reported that physiotherapistsbelieved they increased strength and flexibility when treating PFPS patients [1]. The feasibility study which aimed to show the physiotherapy effect, showed no improvement of strength and flexibility but there was improvement of pain and function. The hypothesis was rejected because when the physiotherapists were asked about how they wanted to see their patients after a six-week treatment, they replied that they aimed to see their patients with less pain, better control and back to their activities only. However, physiotherapists reported that the way patients appear after treatment, also depends on the patients' characteristics and what they want to achieve.

\section{Pain and function improvement}

Physiotherapists reported that strength and flexibility are not part of the physiotherapy practice while pain, control and function improvement are.

FG1P2: ...With less pain, good control and be able to do their sports and their activities.

FG2P7:...symptoms to be improved.

FG2P5:... being able to achieve functionally what they want to do.

FG2P4:... also some objective markers to be improved, like the lower limb alignment, control of their muscles, then, it is more unlikely to come back again.

FG2P1:...if this affects their pain levels then that makes us happy too.

\section{Evidence statement 9}

Physiotherapy aims to improve pain and function which is something shown by previous research. Strength and flexibility improvement is not one of their aims.

Hypothesis that practice depends on the patients

This statement mirrors physiotherapists' perception regarding theirapproach to their practice. This appeared to be patient rather thantreatment-specific. Although their evidence statements showed some constant expectations of their treatment (pain and function improvement, better muscle coordination and fire-off); these could be the basic expectations. Analysis shows that physiotherapy practice dependedfirstly on what the problem appears to be in each individual patient but also on what the patient hoped to achieve from their physiotherapy. This explains why pain and inability to perform everyday 
activities functionally comes as first priority. This implies that the patients' characteristics (non-athletic patients and not willing to comply) affect physiotherapy practice significantly.

\section{Practice depends on patients' characteristics}

FG1P1:...I would not necessarily give them flexibility exercises... Researcher: you wouldn't?

FG1P1:... Well, it depends...

FG1P2:...It depends, yeah.

FG1P1:...It is something that I might do or might don't but I think, I firstly would go for the posture.

FG2P6:...this is where your advice needs to change. You get people who need some rest from their activities and others who need to do something active.

FG1 P5:...it depends whether it is too painful to squat... sometimes is a useful exercise and sometimes they find it painful, it depends on what the patients can do as well. So again it depends on your patients and how they present.

\section{Practice depends on what patients want to achieve}

FG2P4:...and a lot of the time they want you to do something around the knee... they do not think it is useful to do other stuff. FG1 P5:...I think it is a little bit different if you work with top elite sport people because the aim is different isn't it?

\section{Evidence statement 10}

Physiotherapy practice aims to increase function and decrease pain and from this point of view the treatment works. Treatment depends on how patients present and what they want to achieve.

Hypothesis that physiotherapists would think about changing their practice

Physiotherapists claimed that they work on getting patients stronger and more flexible; howeverthis was not provedby a feasibility study at the physiotherapy department. Therefore it was hypothesised that physiotherapists might consider changing their practice. However the hypothesis was rejected because, although they appeared to be willing to change (if they were told to), they did not think that they needed to change becausethey believed that what they do works. However, it appeared that physiotherapists knew that there is also a need for improvement in strength and flexibility; in fact they reported that if strength and flexibility were achieved their patients would not have to return after a short period of time.

\section{Treatment works...}

FG1P2:...I do not know...if the outcomes show that patients are feeling better, then that is the main thing isn't it?

FG2P1:...I think we would be opened to try anything...

FG2P7:....well even though we do not increase strength or flexibility we obviously do something and it is definitely worth doing. So maybe the thing about the strength and flexibility is not actually be needed to make difference anyway... ...but long-term effect of the treatment is needed.

FG2P5:...in terms of the demand of the physios these days, I know from back point of view, we do back education classes, that potentially we could group AKP patients into a group and we could get a program with serious strength and stretching for longer term. This will also increase the education effect and the psychological because patients aware that they are not the only one with this problem.

FG2P2:....we can also provide them with tools for longer term, because they tend to come back with the same problem...they do their exercises and when they find that their pain is reduced they stop doing them. They usually come back in six months. So, that would keep the education in long term and make it lifestyle.

\section{Evidence statement 11}

Physiotherapists believe that what they do is effective. However, their treatment has a short-term effect, probably because no change in strength or flexibility was achieved. This contradicts what physiotherapists reported regarding their long-term effect of their treatment. Group classes and longer term-tools might achieve this and reduce the number of patients coming back for more treatment.

Hypothesis that functional improvement was not enough In a previous study [1] physiotherapists reported that they strengthen and increase flexibilityin patients with PFPS. Therefore, the hypothesis that functional exercises would not be enough was generated. The hypothesis was accepted although some physiotherapists reported that low level functional exercises were enough to treat PFPS patients However the participants who initially said that thisstarted to change their mind when they considered that functional exercises would not help their patients in the long term. Patient characteristics and compliance appeared to be a great barrier. This even applied to those who said that they would definitely do both functional and specific exercises for strength and flexibility.

\section{These are illustrated by the following quotes:}

Functional exercises are enough for low level patients but the pain easily comes back.

FG2P2:...I think they need to be patient specific, probably they are OK for lower level patients but for high level patients you might need something much more specific.

FG2P3:...for me I find that general exercises for gluts and quads, like pain free squats often work...and you are not targeting, you are just getting everything...everything going really...

FG1 P2:...as P5 said, when you are getting them to do normal everyday things, standing properly, and walking up and down the stairs, just normal functional things you don't get any overflow because you do not change any of the motor patterns; and they feel easier to do the exercises, but the pain comes back when this stops.

\section{Functional exercises are not enough}

FG2P6:... I you have assessed a specific problem you cannot leave 
Papadopoulos et al., Physical Therapy and Rehabilitation 2017,

http://www.hoajonline.com/journals/pdf/2055-2386-4-9.pdf

doi: 10.7243/2055-2386-4-9

it aside can you? You need to strengthen it back up.

FG2P5:...both of them (function and specific). And also you need to improve the endurance of the muscles. Not just the strength. Because if the problem comes after 20 minutes of run, then you need endurance and not just strength.

FG1P1:...I might do both (function and specific), it depends on the patient and what you can get them to do. But I think sometimes if you get them to do quads is good because it is a start to wake everything up again...but then again...it depends on the patient really.

\section{Evidence statement 12}

Functional exercises are not enough. The prescription of functional exercises alone depends on the patient characteristics (not motivated patient) and affects long-term physiotherapy results.

\section{Hypothesis that physiotherapists would have to change their exercise prescription}

This code reflects physiotherapists' perception regarding whether they need to change their exercise prescription and prescribe more specific exercises which would have more impact in strength and flexibility. Before the conduction of this study it was believed that physiotherapists would decide to change their prescription in order to restore strength and flexibility. However the hypothesis was rejected because all physiotherapists replied negatively. Physiotherapists thought that the limited time they had to treat each patient was not nearly enough to explain complicated exercises. Many of their patients are non-athletic and physiotherapists reported that the more non-athletic the patient was, the more time they needed for education and exercise explanation.

FG2P5: ... and because we do not have much time per patient we might not be able to explain the exercises as much as the patients need to understand them. We only have 20 minutes on our patients and there is no much time to explain everything. In these 20 minutes there is not enough time to make a difference in muscles and also explain to the patients what they have to do at home.

FG1P2:...I think you should probably progress slower with these people, you might have to see them more often to make sure they comply, because the compliance would be more difficult; and you need more time with them for education.

FG1P2:...I think we do not get them to see them enough.. you know, you assess them one day and the next appointment maybe after a month...you send them away...you have taught them the exercises and given pictures with the exercises and after a month they come back and you notice they have been doing them wrong. And then you are like ohhh...

\section{Evidence statement 13}

Physiotherapists would not change their exercise prescription because specific exercises take time and they only have a little time with patients to explain them. Specific exercises would make patients comply even less and take time from education.

\section{Hypotheses and explanations}

The next step of Ritchie and Spencer, [9] is charting. In this stage the themes are presented in a chart according to the appropriate part of the prior framework to which they relate. Figure 2 shows the merged explanations for each hypothesis.

\section{Evidence statement and level of consensus}

For each of the 13 evidence statements reported by the merger of the answers to the hypotheses, a level of consensus was described using the moderator's notes. Table 2 shows the evidence statements and the level of consensus.

\section{Discussion}

PFPS is a very challenging syndrome especially when it comes to assessing and treating [15]. Because of this, NWW physiotherapists reported that they useda variety of ways to treat the syndrome with the strengthening and stretching of several lower limb muscles among the most important (appendix 2). However, a six week physiotherapy treatment did not significantly improve the strength of any of the lower limb muscles (appendix 1). This study explains the reason that this occurred and the complexity of NHS physiotherapy treatment.

Physiotherapists were not surprised with the results of the feasibility study (appendix 1)because of the type of patients they are dealing with in the NHS. Patients with PFPS who attend the physiotherapy departments of NWW generally do not have the same characteristics or the same needs as those patients described in the literature. Few NHS patients are athletic and according to the physiotherapists they do not get knee pain because of overuse but because of the lack of exercise. These patients are not used to performingexercises; therefore, physiotherapists have to address the patients' reluctance to do prescribed exercises for strength and flexibility. Because of these patient characteristics, physiotherapists only prescribed simple exercises which patients would perform at home during their everyday activities. These exercises, along with the given education, resulted in improvement in pain and function after a six week physiotherapy treatment. Physiotherapists admitted that this kind of treatment may not be enough to induce a physical change because without an increase in strength and flexibility, their treatment will often only have a short period effect with the consequence that some patients come back after a few months. This reflectsthe experience of Noehren et al. [16] who reported that 5 years after rehabilitation $80 \%$ of the patients still report pain. In spite of this, physiotherapists believed in a way their treatment worked and did not feel they needed to change their practice. A major factor here was that because NHS treatment time is so limited, teaching patients how to perform specific complicated exercises for strength and flexibility would leave even less time for education as well as increasing the risk of low compliance. Each of the 13 evidence statements is discussed below, while 
Papadopoulos et al., Physical Therapy and Rehabilitation 2017,

http://www.hoajonline.com/journals/pdf/2055-2386-4-9.pdf

Table 2. Evidence statements and level of consensus.

Evidence statement
Evidence statement 1: Physiotherapists did not deny that their practice aimed to increase strength
and flexibility. However, a six-week time period was not enough to demonstrate improvement in
strength and flexibility. Additionally, they reported that if patients were measured later than those
six weeks, those results would probably be significant. This shows that physiotherapists believed
that their treatment has a long term effect which lasts even after patients are discharged from the
physiotherapy department.

Assistant moderator's notes. Level of agreement

High level-11/12

Evidence statement 2: NHS physiotherapists do not have the same perception as physiologists/sport Moderate level 9/12 scientists regarding how strength and flexibility can be achieved. Physiology evidence shows that an increase in muscle strength can be achieved in six weeks if intense and precise strength and flexibility programmes are applied 3 times per week or in a day by day basis.

Evidence statement 3: It is not a typical physiotherapy NWWpractice to measure strength and flexibility. This evidence shows that exact level of these two characteristics was not intended to be achieved.
Evidence statement 4: Physiotherapists have to deal with non-athletic patients. This kind of patients are not familiar with sport activities, thus physiotherapists prescribe simple and generic exercises (otherwise patients will not perform them) which may lead to a non-significant increase of strength and flexibility. This contradicts the first evidence statement according to which a longer time period would change patients' strength and flexibility

Evidence statement 5: Patients' perceived lack of compliance with prescribed exercises affects physiotherapy practice by inducing physiotherapists to prescribe a few and simple exercises. This may lead to a non-significant increase of strength and flexibility at 6 weeks. Evidence statement 6: Education (psychological effect, knowledge and reassurance and self-
managing) plays an important role in reducing patients' pain and improving function, enhancing
patient compliance and keeping patients without pain long-term.

Evidence statement 7: Functional exercises/inducing patients to do any activity are suitable for the type of patients physiotherapists see in the clinic and play an important role in reducing patients' pain and improving function; however they do not increase strength and flexibility.

Evidence statement 8: A variety of different treatment modalities are used to increase function and
decrease pain. They are all patient-dependent. Focusing on these components rather than active treatments may have played an important role in not increasing strength and flexibility however; all patients do not need better strength and flexibility.

Evidence statement 9: Physiotherapy aims to improve pain and function which is something shown by previous research. Strength and flexibility improvement is not one of their aims.

Evidence statement 10: Physiotherapy practice aims to increase function and decrease pain and from this point of view the treatment works. Treatment depends on how patients present and what they want to achieve.
Evidence statement 11: Physiotherapists believe that what they do is effective. However, their treatment has a short-term effect, probably because no change in strength or flexibility was achieved. This contradicts what physiotherapists reported regarding the long-term effect of their treatment. Group classes and longer term-tools might achieve this and reduce the number of patients coming back for more treatment.

Evidence statement 12: Functional exercises are not enough. The prescription of functional exercises alone depends on the patient characteristics (not motivated patient) and affects long-term physiotherapy results.

Evidence statement 13: Physiotherapists would not change their exercise prescription because precise exercises take time and they only have a little time with patients to explain them. Precise exercises would make patients comply even less and take time from education.

barriers to clinical practice and the lack of fidelity with the literature are described.

\section{Evidence statement 1}

The first high-level evidence statement contradicts what literature suggests regarding strength and flexibility recovery and what physiotherapists reported later on in the interviews.
Physiotherapists said that a longer period of time might have revealed better results, implying that an average of a six-week period is not enough for muscles to get stronger and more flexible and that their treatment would have a long-term effect even after patients got discharged. Literature [17] and bibliography [18] suggest that six weeks can be enough if intense and specific strength and flexibility programs are 
applied 3 times per week or in a day by day basis using the 10 Repetition Maximum (RM) (for strength) or continuous passive stretch for 30 seconds and Proprioceptive Neuromuscular Facilitation (PNF) stretches. The second contradiction is that physiotherapists reported later in the interviews (evidence statement 11) that just because their treatment has a shortterm effect, some patients return to physiotherapy after a few months. There is a need for long term effective treatment because Noehren et al. [16] report that $80 \%$ of their patients still had the same symptoms after 5 years of rehabilitation.

\section{Evidence statement 2}

This second moderate-level evidence statement was about the perception that NHS physiotherapists had regarding strength and flexibility. Most of the physiotherapists $9 / 12$ reported that they would increase strength and flexibility with functional exercises such as stair descents/small knee bends. This contradicts what physiologists or sport scientists have in mind when they think about these two characteristics. According to Kroemer [19] strength is the maximal force a muscle can exert isometrically in a single voluntary effort, whilst, flexibility is the amount of movement of a joint through its normal plane of motion [20]. These two muscle characteristics do not increase with functional exercises but with intense methods described in the previous evidence statement.

\section{Evidence statement 3}

The third moderate-level evidence statement shows another contradiction. When it was proposed whether NHS physiotherapists need a better way to assess strength and flexibility most of them 10/12 were positive. However, after a few minutes of conversation they realised that it is not usual practice to measure strength and flexibility. This situationis in conflict with the regulations of the HCPC standards of physiotherapy proficiency which suggest the use of appropriate outcome measures on every occasion [2].

\section{Evidence statement 4}

This high-level evidence statement brought to light an important lack of fidelity between literature and clinical practice. Most reported studies recruited athletic populations [14]; hence, the evidence is based on patients whose PFPS was associated with overuse or other athletic reasons. NWW physiotherapists reported that most of their patients (about $80 \%$ ) were not athletic and in these cases the syndrome occurred because of lack of activity. This lack of fidelity between the literature and clinical practice is not appreciated. In fact Lankhorst et al. [21] suggested that more research should be conducted in athletic population. This will not be applicable to NHS clinical practice. The second component of this evidence statement is that because patients are not athletic they are not keen on specific exercises that physiotherapists wanted to prescribe; therefore, physiotherapists only give simple exercises which aim to restore function ability. The last statement contradicts evidence statement 1 according to which physiotherapists reported that their treatment might restore patients' strength and flexibility if more than 6 weeks were given.

\section{Evidence statement 5}

This fifth high-level evidence states that according to NWW physiotherapists, patients with PFPS do not comply with the prescribed exercises. This behaviour is characteristic of non-athletic people when it comes to exercise. Consequently physiotherapists prescribe simple and interesting exercises that patients can perform in the course of the daily round. If the exercises did not have these characteristics patients would not perform any of them. This evidence may explain why strength and flexibility was not achieved but shows once again the lack of fidelity between literature, which suggests the focusing of specific exercises, and the clinical practice which gets patients to perform simple and functional exercises.

\section{Evidence statement 6}

This high-level statement showed the importance of education in managing PFPS. According to what the NWW physiotherapists report, education can be separated in to three categories; psychological effect, knowledge and reassurance and self-managing. Participants believed that physiotherapy has a psychological effect by supporting patients with PFPS and listening to their problem. This is in line with the findings of Lankhorst et al. [21] according to which 'looking for social support' was a significant risk factor for patients with PFPS. Additionally, explanation about the cause of the painful knee and what the patient can do about it, along with reassurance that it is not a major problem and that surgery is not required seemed to help patients decrease their pain levels. This is in line with the HCPC standards of physiotherapy proficiency according to which psychosocial, social and cultural factors that influence an individual in health and illness must be understood and supported by physiotherapists [2]. Selfmanagement is an important outcome of patient education. This makes it easier for patients to deal with their condition so that it causes them less distress and disability. Another benefit of successful self-management is a reduction in re-referral for further physiotherapy for the same complaint. Many patients continue to have some pain but have learned to accept it and that manage it themselves.

\section{Evidence statement 7}

This high level statement shows that NWW physiotherapists chose deliberately to prescribe functional exercises or any sporting activity than specific exercises for strength and flexibility. This evidence shows that there is a lack of fidelity between the literature and clinical practice since literature showed specific single exercises(single leg squat) for several lower limb muscles such as quadriceps and hip abductors [22]. However, evidence statement 4 showed that physiotherapists have to deal with patients who have PFPS because they do 
not do any activity. Specific exercises are often unsuitable for these non-athletic patients who have high levels of noncompliance. In fact physiotherapists reported that it is a challenge to get these patients do any activity. Apart for the three education categories reported in evidence statement 6 , patients' education should also include the importance of specificexercises in PFPS treatment.

\section{Evidence statement 8}

This high-level evidence statement reports, that physiotherapists use most of the treatment componentssuggested in the literature to improve function and pain and demonstrates that they are aware of the cutting-edge physiotherapy for PFPS. Additionally, physiotherapists said in the interviews that not all PFPS patients need strengthening and stretching which is acceptable since not all risk factors are related to muscle weakness or stiffness [21].

\section{Evidence statement 9}

This high-level evidence shows that NWW physiotherapy for PFPS aims to improve pain and function but not strength and flexibility. However, the HCPC standards of physiotherapy proficiency (2013) do not include strength and flexibility in the physiotherapy treatment. Therefore, apart from the confusion regarding what strength and flexibility is and how improvements therein can be achieved (evidence statement 2), there was inconsistency regarding how useful these two muscle characteristics are in treatment. At the beginning of the interviews they reported that strength and flexibility would be achieved if more time was given to muscles, then after consideration they said that they do not aim to restore these muscle characteristics and later on they accepted that if improvement in strength and flexibility were achieved, it might prevent some patients returning for further treatment.

\section{Evidence statement 10}

The tenth high-level evidence statement shows that NWW physiotherapists aim to improve pain and function and appendix 1 revealed that this was achieved. This is in keeping with the HCPC standards of physiotherapy proficiency report that NHS physiotherapists should implement and manage physiotherapy interventions aimed at the facilitation and restoration of movement and function [2]. Additionally, their physiotherapy practice is patient-specific and dependent on what patients want to achieve. This was explained in a previous study [1] where physiotherapists reported that they do not treat syndromes but patients' needs. Patients seek help when they are in pain or cannot perform everyday activities.

\section{Evidence statement 11}

As reported in evidence statement 10, PFPS physiotherapy treatment works; not through lower limb strength and flexibility improvement but through improving quality of movement and restoration of function. Physiotherapists appeared to acknowledge that some of their patients are re-referred to the physiotherapy department after a few months with the same symptoms. This shows that in some cases their treatment has a short-term effect which partly contradicts what physiotherapists reported in evidence statement 1. Physiotherapists reported that group classes in the physiotherapy department might help in strength and flexibility improvement because patients would be supervised and all have the benefits of group dynamics. Group treatment has been tested with low back pain patients in NWW physiotherapy department and according to the physiotherapists they have been successful in pain and function improvement.

\section{Evidence statement 12}

This moderate-level evidence statement confirms that most of the NWW physiotherapists know that what they and their patients do is not enough to increase strength and that their practice as it is often has no physical long-term effect. However, physiotherapists believed that the barriers they have to confront (patient compliance and time per patient treatment) cause this kind of practice. Therefore, since there is no 'ideal world' the long-term effect of group classes along with more education are the two suggestions that could be tested in the future. Group classes might get patients more active for more time. On the other hand education should include the importance of right exercise for their problem while selfmanaging the pain would make them live with it. Noehren et al. [16] may reported that $80 \%$ of PFPS patients still reported pain after a 5 -year rehabilitation and the pooled data of Collins et al., [23] and van Linschoten et al. [24] $(n=310)$ showed that $40 \%$ of the patients still reported persistent complaints one year post intervention; however, if those patients knew how self-manage their pain and live with it they probably would not have to return for more physiotherapy.

\section{Evidence statement 13}

Because of the previous evidence statements it is not surprising that thephysiotherapists reported that they would not change their practice by prescribing specific exercises to their PFPS patients. According to physiotherapists, specific exercises take time, cause patients to comply even less and reduce the time available for the all-important education. This evidence statement shows the gap between the clinical practice and literature based on athletic patients and applied in physiology laboratories.

\section{Conclusions}

This study has shown the lack of fidelity between literature and clinical practice. According to the physiotherapists, the available literature is based on athletic patients with PFPS, while patients who are referred in the NHS clinic of NWW district hospital are largely non-athletic. The specific exercises are not effective because they are not used by NWW physiotherapists. The exercise-based interventions suggested by the literature 
are not adoptable in a NHS environment because of several barriers. Given the lack of evidence for the patients they see, NWW physiotherapists aim to improve pain and function and in that way their treatment works. However, this practice often only has a short-term effect. Future research should focus on non-athletic patients with PFPS and the new evidence should be applicable to the challenging environment of NHS clinics. Finally, group classes and better education on the importance of specific exercises and self-managing should be researched whilst the long-term effect of these treatment components should also be assessed.

\section{Additional files}

\section{Appendix 1 \\ Appendix 2}

\section{Competing interests}

The authors declare that they have no competing interests.

\section{Authors' contributions}

\begin{tabular}{|l|c|c|c|}
\hline Authors' contributions & KP & RK & DS \\
\hline Research concept and design & $\checkmark$ & -- & -- \\
\hline Collection and/or assembly of data & $\checkmark$ & -- & -- \\
\hline Data analysis and interpretation & $\checkmark$ & -- & -- \\
\hline Writing the article & $\checkmark$ & -- & -- \\
\hline Critical revision of the article & $\checkmark$ & $\checkmark$ & $\checkmark$ \\
\hline Final approval of article & $\checkmark$ & $\checkmark$ & $\checkmark$ \\
\hline Statistical analysis & -- & -- & -- \\
\hline
\end{tabular}

\section{Acknowledgement}

The authors would like to thank the NWW physiotherapists who took part in this study.

Publication history

Editor: Catherine Ortega, University of Texas Health Science Center, USA.

Received: 05-Jun-2017 Final Revised: 07-Aug-2017

Accepted: 22-Aug-2017 Published: 09-Sep-2017

\section{References}

1. Papadopoulos K.D, Noyes J, Barnes M, Jones J.G and Thom J.M. How do physiotherapists assess and treat Patellofemoral Pain Syndrome in North Wales? A mixed method study. (2012) International Journal of Therapy and Rehabilitation. 2012; 19:261-272. | Article

2. Health \& Care Professions Council. Standards of proficiencyphysiotherapists. Publication code: 20130301jPOLPUB. 2013.

3. Papadopoulos K.D and Kabir R. Physiotherapy effectiveness on muscle strength, flexibility, pain and function in patients with patellofemoral pain syndrome. 2016; Journal Of novel Physiotherapy and Physical Rehabilitation. 3: 035-039.

4. Kitzinger J. Qualitative research. Introducing focus groups. BMJ. 1995; 311:299-302. | Article | PubMed Abstract | PubMed FullText

5. Onwuegbuzie A.J, Dickinson W.B, Leech N.L and Zoran A.G. A Qualitative Framework for Collecting and Analyzing Data in Focus Group Research. International Journal of Qualitative Methods. 2009; 8:1-21. | Article

6. Johnson R.B and Christensen L.B. Educational research: Quantitative, qualitative, and mixed approaches. Boston, US: Allyn and Bacon. 2004.

7. Krueger R.A. The methodology of focus groups: The importance of interaction between research participants. Sociology of Health and IIIness. 1994; 18:103-121. | Article
8. Morrison-Beedy D, Cote-Arsenault D and Feinstein NF. Maximizing results with focus groups: moderator and analysis issues. Appl Nurs Res. 2001; 14:48-53. | Article | PubMed

9. Ritchie J and Spencer L. Qualitative data analysis for applied policy research. Analysing Qualitative Data.London, UK: Routledge. 1994.

10. Carr A, Hewlett S, Hughes R, Mitchell H, Ryan S, Carr M and Kirwan J. Rheumatology outcomes: the patient's perspective. J Rheumatol. 2003; 30:880-3. | Article | PubMed

11. Patton M.Q. Qualitative research and evaluation methods. London, UK: Sage Publications Inc. 2002.

12. Krueger R.A and Casey M.A. Focus groups: a practical guide for applied research. London, UK: Sage Publications Inc. 2000.

13. Heidegger M. Being and time. London, UK: SCM Press Ltd. 1962.

14. Papadopoulos K.D, Stasinopoulos D and Ganchev D. A systematic review of systematic reviews of PFPS risk factors, diagnostic tests, outcome measures and exercise treatment, with an analysis of key participant and study characteristics in included primary studies. The Open Sports Medicine Journal. 2015; 9:7-17. | Pdf

15. Collins NJ, Bisset LM, Crossley KM and Vicenzino B. Efficacy of nonsurgical interventions for anterior knee pain: systematic review and meta-analysis of randomized trials. Sports Med. 2012; 42:31-49. | Article | PubMed

16. Noehren B, Scholz J and Davis I. The effect of real-time gait retraining on hip kinematics, pain and function in subjects with patellofemoral pain syndrome. Br J Sports Med. 2011; 45:691-6. | Article | PubMed

17. Micheo $W$, Baerga $L$ and Miranda $G$. Basic principles regarding strength, flexibility, and stability exercises. PM R. 2012; 4:805-11. | Article | PubMed

18. Kisner $C$ and Colby A.L. Therapeutic exercise: foundations and techniques, sixth edition. Philadelphia, US: F.A. Davis Company. 2012.

19. Kroemer $\mathrm{KH}$. Human strength: terminology, measurement, and interpretation of data. Hum Factors. 1970; 12:297-313. | Article | PubMed

20. Knapik JJ, Bauman CL, Jones BH, Harris JM and Vaughan L. Preseason strength and flexibility imbalances associated with athletic injuries in female collegiate athletes. Am J Sports Med. 1991; 19:76-81. | Article | PubMed

21. Lankhorst NE, Bierma-Zeinstra SM and van Middelkoop M. Risk factors for patellofemoral pain syndrome: a systematic review. J Orthop Sports Phys Ther. 2012; 42:81-94. | Article | PubMed

22. Heintjes E.M, Berger M, Bierma-Zeinstra S.M.A, Bernsen R.M.D, Verhaar J.A.N and Koes B.W. Exercise therapy for patellofemoral pain syndrome. Cochrane Database of Systematic Reviews. 2009; 4:CD003472.

23. Collins N.J, Crossley K.M, Beller E, Darnell R, McPoil T and Vicenzino B. Foot orthoses and physiotherapy in the treatment of patellofemoral pain syndrome: randomised clinical trial. British Medical Journal. 2008; 337. | Article

24. van Linschoten $R$, van Middelkoop $M$, Berger MY, Heintjes EM, Verhaar $J$ A, Willemsen SP, Koes BW and Bierma-Zeinstra SM. Supervised exercise therapy versus usual care for patellofemoral pain syndrome: an open label randomised controlled trial. BMJ. 2009; 339:b4074. | Article | PubMed Abstract | PubMed FullText

\section{Citation:}

Papadopoulos K, Kabir R and Stasinopoulos D.

Perceptions of physiotherapists of their role in reducing pain and increasing, function, strength and flexibility in patients with Patellofemoral Pain Syndrome. Phys Ther Rehabil. 2017; 4:9.

http://dx.doi.org/10.7243/2055-2386-4-9 